Article

\title{
Insights into the Variations of Hao-Dependent Nitrifying and Nir-Dependent Denitrifying Microbial Communities in Ammonium-Graduated Lake Environments
}

\author{
Ruojin Zhao ${ }^{1,+}$, Yinyan Chen ${ }^{1,+}{ }^{,}$Jin $Q u^{1}$, Peng Jin ${ }^{2, *} \mathbb{D}$, Zhanwang Zheng ${ }^{1,3, *}$ and Zhiwen Cui ${ }^{3}$ \\ 1 The Key Laboratory for Quality Improvement of Agricultural Products of Zhejiang Province, \\ The College of Agricultural and Food Sciences, Zhejiang A \& F University, Hangzhou 311300, China \\ 2 School of Environmental \& Resource, Zhejiang A \& F University, Hangzhou 311300, China \\ 3 Zhejiang Shuangliang Sunda Environment co., LTD, Hangzhou 310000, China \\ * Correspondence: jinpeng@zafu.edu.cn (P.J.); Zwzheng@zafu.edu.cn (Z.Z.) \\ + These authors contributed equally to this work.
}

Received: 8 July 2019; Accepted: 6 August 2019; Published: 7 August 2019

check for updates

\begin{abstract}
Biological nitrification and denitrification play significant roles in nitrogen-associated biogeochemical cycles. However, our understanding of the spatial scales at which microbial communities act and vary is limited. We used gene-specific metagenomic PCR to explore changes in nitrifying and denitrifying microbial communities within pristine lake and its branches, where the ammonium and dissolved organic carbon (DOC) concentrations form a gradient. The biomarkers hydroxylamine oxidoreductase and nitrite reductase genes indicated that strong relationships exist between the diversities and community structures of denitrifiers and ammonium gradients. It showed that the Nitrosomonas oligotropha cluster dominates the nitrifying bacteria in low-nutrition environments, while a new Nitrosomonas ureae cluster accounted for nearly $80 \%$ of the nitrifying bacteria in high-nitrogen environments. The distribution and diversity of nirS/K-dependent denitrifiers in the various habitats were similar, but predominantly affiliated with unknown clusters. Moreover, the abundance of all the hao genes dramatically outnumbered that of nir genes. The relative abundance of hao was clearly higher during eutrophication (13.60\%) than during oligotrophy (5.23\%), whereas that of nirS showed opposite tendencies. Overall, this study provides valuable comparative insights into the shifts in nitrifying and denitrifying microbial populations in lake environments with ammonium gradients, suggesting that unique dominant denitrifiers probably play an important role in the nitrogen cycle.
\end{abstract}

Keywords: microbial diversity; hydroxylamine oxidoreductase; nitrite reductase; ammonium gradient; metagenomic; denitrifiers

\section{Introduction}

The global biogeochemical nitrogen cycle has undergone dramatic changes in the past few decades. In particular, anthropogenic processes, including agricultural, industrial, and domestic activities, have resulted in an unprecedented increase in environmental nitrogen release. Overloading terrestrial ecosystems with ammonium, nitrate, and nitrite as the principal nitrogen pollutants has serious negative effects on human health, biodiversity, and water quality [1]. The coupled nitrification-denitrification and nitrification-anaerobic ammonium oxidation (anammox) processes mediated by environmental microorganisms are known to play important roles in the transformation and biogeochemical cycling of nitrogen, and probably contribute to the removal of up to $50 \%$ of the external dissolved inorganic 
nitrogen that enters the ecosphere [2,3]. Environmental conditions (i.e., carbon or nitrogen) can significantly influence the community structures and relative abundances of microorganisms [4]. The abilities of particular lineages of organisms to survive in specific environments may reflect relevant aspects of environmental nutrient availability $[5,6]$. The diversity and structures of bacterial communities in lakes, estuaries [7], sea coasts [8,9], and wastewater treatment plants [10-12] have been extensively investigated. Most studies of community bacterial phylogenies from single-source environmental samples have been based on the conventional analysis of 16S rRNA genes or Denaturing gradient gel electrophoresis (DGGE) analysis of specific genera [13]. However, the great divergence among different bacterial taxa is not directly related to the major environmental factors. Thus, it is important to investigate the variations and shifts in community composition regulated by the aquatic environmental factors [14,15].

To date, there has been few investigations available on the comparative analysis of microorganisms in lake environments where nutrients vary greatly within the same region, especially those involving nitrogen metabolisms [7]. The lake reservoir is influenced by the dynamic inflow tributaries adjoining residential areas, and may serve as a transitional zone from the eutrophication phylotypes of nitrifying and denitrifying bacteria to the oligotrophication ones. Therefore, a refined and targeted comparative description of the diversity of nitrifying and denitrifying communities in freshwater lakes with ammonia gradients is required in order to improve our understanding of the shifts in the nitrifying and denitrifying populations that occur with sharp differences in the environmental nitrogen status. This should also offer an opportunity to identify populations that are specific to those environments.

The objectives of the present research were to investigate the pronounced community shifts in nitrifying and denitrifying populations in different environments (where there are sharp differences in the nitrogen and organic carbon status). A gene-specific metagenomic targeted PCR approach [16] was employed to comparatively characterize the nitrifying and denitrifying microbial communities from three environmental waters with different hydrological features, trophic statuses, and nitrogen pollution levels (Table 1). We expected that differences in the structures of nitrogen-transforming communities would accompany differences in nitrogen availability among three samples. We tested this by sequencing the hydroxylamine oxidoreductase (HAO) and nitrite reduction (copper-containing NirK and cytochrome cd1-containing NirS)-encoding genes of representative nitrifying and denitrifying bacteria within these samples, and compared their relative abundances with qPCR. These findings should extend our understanding of the bacterial communities that inhabit aquatic ecosystems and may have utility in the sustainable management of water resources.

Table 1. General physicochemical parameters of three environmental waters of the pristine Qingshan Lake and its branch, the Sewage River.

\begin{tabular}{cccccccc}
\hline & $\mathrm{TN}$ & $\mathbf{N H}_{4}{ }^{+}-\mathbf{N}$ & $\mathbf{N O}_{3}{ }^{-}-\mathbf{N}$ & $\mathbf{N O}_{2}{ }^{-}-\mathbf{N}$ & $\mathbf{C O D}$ & $\mathbf{p H}$ & DO \\
\hline QL-H & 0.21 & 0.08 & 0.06 & $\mathrm{ND}^{\mathrm{a}}$ & 13 & 6.83 & 4.91 \\
QL-V & 16.82 & 14.36 & 1.15 & 0.14 & 75 & 6.97 & 5.71 \\
SR-W & 121.32 & 93.41 & 5.67 & 0.87 & 331 & 7.71 & 3.35 \\
\hline
\end{tabular}

${ }^{a}$ ND not detectable, unit $\mathrm{mg} / \mathrm{L}$.

\section{Materials and Methods}

\subsection{Sample Collection and Chemical Analyses}

The Qingshan Lake reserve watershed area is approximately $620 \mathrm{~km}^{2}$, and is the important potable water sources of Lin'an city. There are mainly six tributaries adjoining the residential area and inflowing to the Western basin of the lake. Three samples representing the three main nutrient levels (i.e., ammonium; total nitrogen, $\mathrm{TN}$; and chemical oxygen demand, COD) were collected in Qingshan Lake from the tributary water to the reservoir area. All the sludge samples were collected from the surface sediments $(5 \mathrm{~cm})$ at a water depth of 30 to $50 \mathrm{~cm}$. High-quality water samples of Qingshan 
Lake (QL-H) and those closest to the village of Qingshan Lake (QL-V) were taken from a reservoir area and the adjoining residential area, respectively. The other Sewage River sample (SR-W) was taken from a domestically polluted tributary. A sample was taken by collecting at least six individual samples at the same depth in the same area to form a composite sample in the study. Metagenomic DNA was isolated from the sludge samples with the Mo Bio PowerSoil ${ }^{\circledR}$ DNA Isolation Kit (Mo Bio Laboratories, Inc., Carlsbad, CA, USA). To remove any coextracted humic substances and other contaminants, the metagenomic DNA was further purified and concentrated with ethanol precipitation, as previously described [17].

The dissolved oxygen (DO) concentrations were measured with the Winkler method [18]. The concentrations of ammonium, nitrate, and nitrite were determined with Nessler's reagent spectrophotometry at $420 \mathrm{~nm}, \mathrm{~N}$-(1-naphthalene)-diaminoethane spectrophotometry at $410 \mathrm{~nm}$, and ultraviolet (UV) spectrophotometry at $540 \mathrm{~nm}$, respectively. Total nitrogen (TN) was measured with the standard UV spectrophotometry method (DR6000, Hach, Loveland, CO, USA).

\subsection{Primer Design and PCR Amplification}

Over 500 nucleotide and protein sequences of the hydroxylamine oxidoreductase (HAO) and nitrite reductase (NirS/NirK) genes were retrieved from the NCBI database (https://www.ncbi.nlm. nih.gov/protein/) with an identity $>30 \%$ and size $>500$ b.p. Then, all the collected protein sequences were performed to multiple sequence alignment analysis and phylogenetic tree construction using the online tool Clustal Omega (https://www.ebi.ac.uk/Tools/msa/clustalo/). Then, some representative sequences from the sub-branch were selected to design gene-specific degenerate primers and phylogenetic analysis. Primers JBHAO-170F (GTATGAVGCGYTGGTNAAGCGYTA), JBHAO-939R (TGGAACTGGRAHGTHCVTCTCAAG), JBNirS-1001F (CGTGGTGGGAAAYTAYTGGCCKCC), JBNirS-1242R (CAYGAYGGHGGHTGGGAC), JBNirK-526F (CACGACGCTCACGGNATGTAYGG), and JBNirK-1002R (GTGCGCGACARCGCRTGRTCNAC) were designed according to the sequence alignments of the typical candidate hao (12), nirS (20), and nirK (19) nucleotide sequences, respectively.

Metagenomic DNA was used for the PCR amplification of 16S rRNA genes (prokaryote specific primers 27F (AGAGTTTGATCCTGGCTCAG) and 1492R (TACGGCTACCTTGTTACGACTT)), nirS, and hao genes. PCR products were separated by agarose gel electrophoresis and cloned into the pMD19-T vector (TaKaRa, Bio Inc., Shiga, Japan) according to the manufacturer's instructions. Randomly selected clones from each sample were sequenced by Shanghai Sangon Biological Engineering Technology \& Services Co. Ltd., China.

\subsection{Quantitative PCR Analysis of hao, nirS, nirK, and $16 S$ rDNA Genes}

To determine the gene abundances in $1 \mathrm{ng}$ of extracted DNA, quantitative PCR (qPCR) was performed in a Corbett Real-Time PCR Machine with the Rotor-Gene 6000 series software 1.7 (Qiagen, Netherlands), using the SYBR Green. The abundances of nitrifiers and denitrifiers were measured by quantifying the hao, nirS, and nirK genes. The abundances of microbial communities were determined by quantifying the $16 \mathrm{~S}$ rRNA gene. Standard curves were constructed for the hao, nirS, and nirK genes using the positive recombinant plasmids Hhao-05/pMD19, HnirS-4/pMD19, and VnirK-15/pMD19 as standards, respectively. For the $16 \mathrm{~S}$ rRNA gene, the positive recombinant plasmid W16S-1/pMD19 was used as the standard. The samples and standards were analyzed with qPCR in triplicate, and the specificity of the qPCRs was confirmed with a melting curve analysis, agarose gel electrophoresis, and DNA sequencing. The efficiencies of the PCRs were 86.9-98.7\%, with $R^{2}$ values $>0.993$ for all the calibration curves.

\subsection{Phylogeny Analysis}

Positive clones were randomly selected and sequenced using universal primers M13. DNA sequences were analyzed using the BLASTN tool, and amino acid sequences were analyzed using BLASTP (https://blast.ncbi.nlm.nih.gov/Blast.cgi) to aid the selection of the closest reference sequences. 
To determine the evolutionary relationship of these environmental Nir and HAO enzymes with established nitrifying and denitrifying microbial communities, the sequences were compared to representative Nir and HAO enzymes from the non-redundant (NR) protein sequence database (NCBI), using neighbor-joining phylogenetic analysis. One thousand bootstrap replications were performed using the MEGA software (MEGA 5.0) [19].

\subsection{Nucleotide Sequence Accession Numbers}

Partial 16S rRNA gene sequences were deposited under genbank Accession No MH155326-MH155426, while partial hao, nirS, and nirK gene sequences were deposited under Accession No MH156255-MH156333, MH156334-MH156390 and MH156391-MH156420, respectively.

\section{Result}

\subsection{Environmental Characteristics}

To determine the relevance of the inorganic nitrogen concentration in the water to nitrifying and denitrifying microorganisms, the physicochemical characteristics of the samples collected in this study were determined and summarized in Table 1 . The collected samples showed significant differences in their concentrations of TN, ranging from 0.21 to $121.32 \mathrm{mg} / \mathrm{L}$. The TN concentrations were significantly higher in the polluted river samples than in the lake samples. Overall, ammonium, nitrate, nitrite, and the chemical oxygen demand (COD) showed distinct gradients, decreasing dramatically lakeward, with ranges of 93.41 to $0.08 \mathrm{mg} / \mathrm{L}, 5.67$ to $0.06 \mathrm{mg} / \mathrm{L}, 0.87$ to below the detection limit, and 334 to 13 , respectively. The river sediments had a higher $\mathrm{pH}$ (7.71) than the lake sediments. The DO concentrations ranged from 3.35 to $5.71 \mathrm{mg} / \mathrm{L}$ in the three environmental points.

\subsection{Distributional Differences in Microbial Communities in Nitrogen-Related Environments}

Analysis of the 16S rRNA gene sequences in the genomic DNA from Qingshan Lake (QL-H and QL-V) and Sewage River (SR-W) are shown in Figure 1. A total of 108 16S rRNA gene sequences were obtained from the environmental sediment samples collected in this study, using 16S rRNA gene-based biomarkers and PCR amplification. A BLAST sequence analysis confirmed that approximately $93.52 \%$ of the amplified sequences (101 sequences) belonged to uncultured bacteria, and that they shared sequence identities of $88-100 \%$ with previously reported $16 \mathrm{~S}$ rRNA sequences. A multiple alignment of all 108 sequences showed that the percentage identities of the nucleotide sequences were $29.14 \%$ to $100 \%$. According to phylogenetic and statistical analyses, the $16 \mathrm{~S}$ rRNA gene sequences were grouped into five distinctive clusters (Figure 1). From this unique dataset, we identified 17 different phyla, with the overwhelming majority of sequences ( $90 \%$ of all sequences) belonging to a few bacterial phyla: Proteobacteria, Aquabacterium, Bacteroidetes, Cytophagales, and unclassified bacteria. More than $90 \%$ of these were uncultured microorganisms, and unclassified bacteria constituted approximately $40-60 \%$ of the sequenced clones. A phylogenetic analysis showed that Proteobacteria comprised $35 \%$ of the total sequences, and comprised an even larger proportion in the 16S rRNA metagenomic clone library, which might truly reflect the community diversity. Proteobacteria was the dominant phylum in all three sludge samples, accounting for about $39.40 \%, 25.81 \%$, and $43.18 \%$ of the phyla in samples QL-H, QL-V, and SR-W, respectively (Figure 1). 


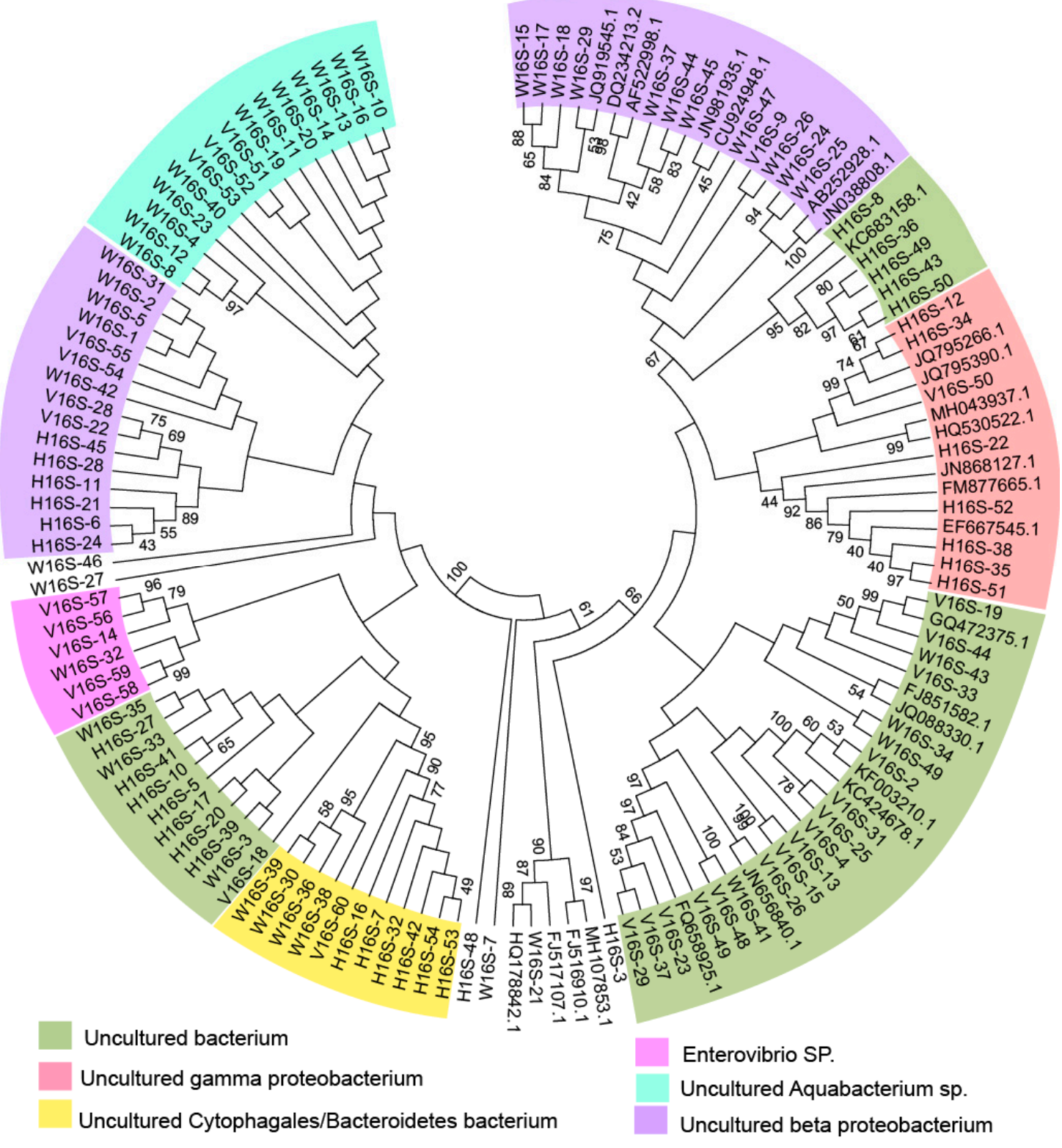

Figure 1. Analysis of microbial communities based on 16S rRNA partial gene sequences retrieved from three environmental collections and other representative organisms' species. Clones H16S, V16S, and W16S were from a high-quality Qingshan Lake water site (QL-H), the Qingshan Lake site closest to the village (QL-V), and domestic pollution in the Sludge River (SR-W), respectively.

\subsection{Phylogenetic Diversity of Hao-Dependent Nitrifying Bacteria in Sediments}

The nitrifying bacterial hao genes in the three samples were amplified from the metagenomic DNA (template) with primers JBHAO-170F and JBHAO-939R to detect the presence of ammonia-oxidizing bacteria (AOB). The PCR products revealed the presence of AOB in these samples (Figure 2). The amplification products were cloned and sequenced, and three clone libraries were constructed. An analysis of randomly selected clones indicated that all the clones of approximately the expected size (817 b.p.) were hao gene fragments, and no non-hao genes were amplified. A total of 132 clones from the three clone libraries were randomly selected and sequenced. The BLASTP results indicated that all the clones contained highly similar hao sequences, with approximately $90-99 \%$ identity to other hao gene sequences in the database. Only 79 novel gene sequences were detected among the sequenced clones, except for the redundant clone sequences. The deduced amino acid sequences of these gene fragments 
matched the conserved regions in the previously characterized functional domains within other Hao proteins. These results demonstrate that the PCR primers that were newly designed to detect hao genes in various sediment samples, JBHAO-170F and JBHAO-939R, are useful for identifying the diversity of $A O B$ nitrifiers with high resolution ( $100 \%$ coverage) and specificity. A multiple alignment of all these sequences showed that the percentage identity between pairs of Hao protein sequences ranged from $82.96 \%$ to $99.63 \%$. The nucleic acid sequences corresponding to these protein sequences showed between $77.90-99.63 \%$ identity.
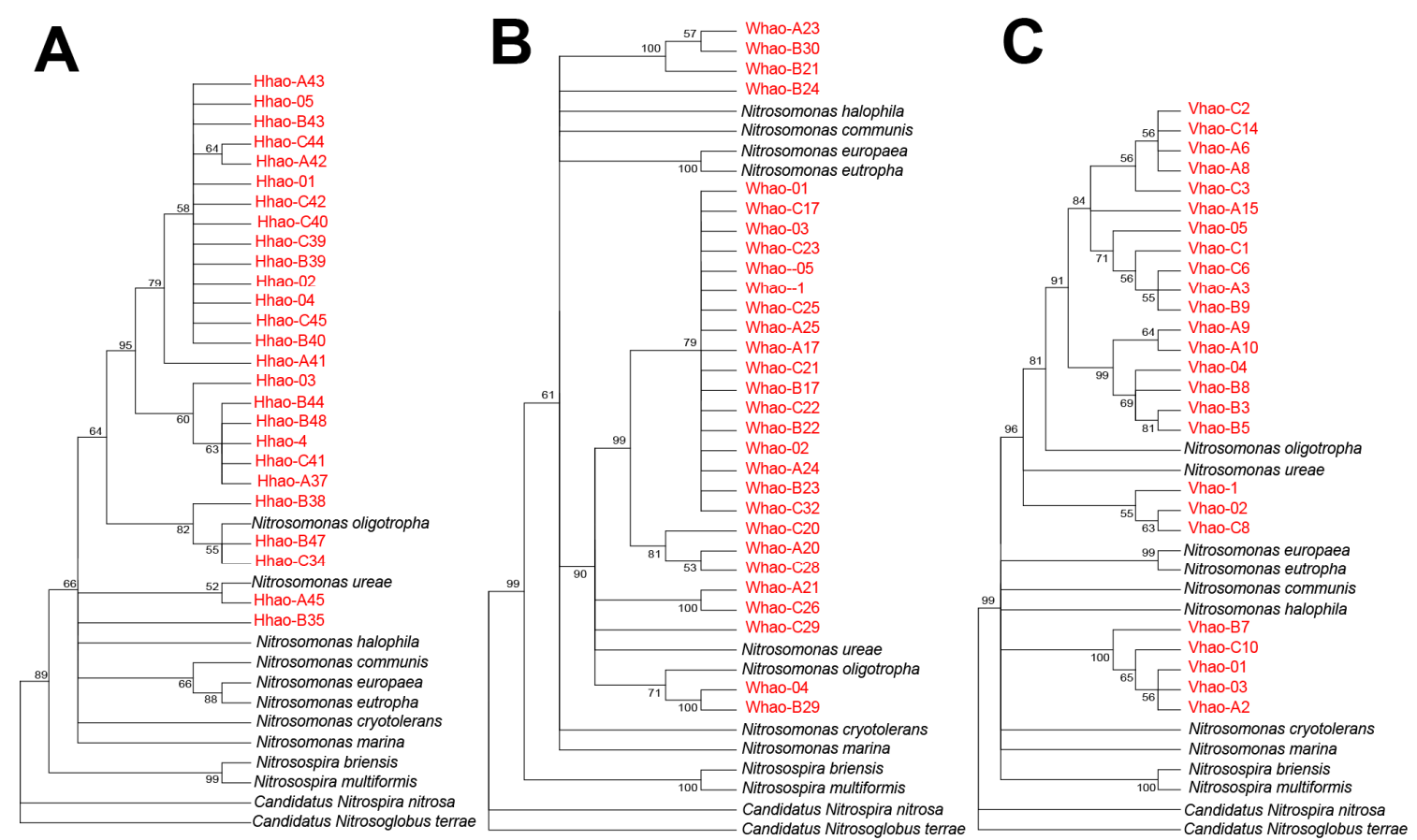

Figure 2. Trees of hydroxylamine oxidoreductase (HAO) constructed based on protein sequences using the neighbor-joining method. Sequences from this study are shown in red (A) sample QL-H; (B) sample SR-W; (C) sample QL-V). Other representative HAO from the protein database and GenBank accession numbers are BAW79844 (Candidatus Nitrosoglobus terrae), CUS31385 (Candidatus Nitrospira nitrosa), WP_074976104 (Nitrosospira multiformis), WP_074793392 (Nitrosospira briensis), SEN74695 (Nitrosomonas marina), WP_074907499 (Nitrosomonas communis), SDZ06451 (Nitrosomonas halophila), U04053 (Nitrosomonas europaea), WP_074929561 (Nitrosomonas eutropha), WP_074202572 (Nitrosomonas cryotolerans), SEG22801 (Nitrosomonas ureae), SDX60482 (Nitrosomonas oligotropha).

The 79 Hao gene sequences were classified based on previous studies by comparing the amino acid sequences of AOB Hao enzymes [20]. As shown in Figure 2A, a phylogenetic analysis of the Hao sequences with the neighbor-joining method showed that the QL-H sample bacterial Hao proteins were affiliated to the Nitrosomonas cluster, whereas no sequence from the Nitrosospira lineage, which dominates high-nitrogen environments, was observed [21]. All of the retrieved QL-H Hao sequences strongly belonged to the $N$. oligotropha cluster and shared between $89.34-99.63 \%$ identity. Only one sequence (Hhao-A45) clustered with Hao of N. ureae, with $91.54 \%$ amino acid sequence identity. However, 17 of the 25 Hao proteins from the QL-V sample were most affiliated with the N. oligotropha cluster and shared $91.18-96.63 \%$ amino acid identity; five proteins fell between the N. communis and $N$. halophila clusters; and three proteins (Vhao-1, Vhao-02, and Vhao-C8) did not match the Hao proteins of these species (Figure 2C). Interestingly, SR-W sample sequences from Sewage River sludge were sporadically affiliated with the subphyla N. communis, N. halophila, and N. oligotropha clusters, but a new cluster accounted for nearly $80 \%$ of the cloned sequences and shared $90.07-99.63 \%$ identity with the closest environmental cluster, N. ureae (Figure 2B). 


\subsection{Diversity of NirS-Dependent Denitrifying Communities}

Nitrite reductase reduces nitrite to nitric oxide in the initial step of two distinct dinitrogen-forming reactions: bacterial denitrification and anammox [2]. They include two structurally different but functionally equivalent forms: copper-containing (NirK) and cytochrome $c d_{1}$-containing (NirS) nitrite reductases [22]. To understand the diversities of the NirK-type and NirS-type denitrifying microbial communities in different environments, the abundances of the nirK and nirS genes in the three samples were detected and quantified with metagenomic qPCR. A total of 131 partial nirS gene sequences were identified, and 57 final unique nirS sequences (74 redundant sequences) were recovered from the sediments of the QL-H, QL-V, and SR-W samples (Figure 3). These results indicated high specificity and sufficient coverage by newly designed primers. These nirS genes shared $76-99 \%$ identity with other nirS sequences in the database. The nucleic acids corresponding to the protein sequences shared relatively high similarities, with approximately $73-100 \%$ identity, to matched nirS sequences in the NCBI database, which were detected in a variety of environmental samples $[8,23]$.

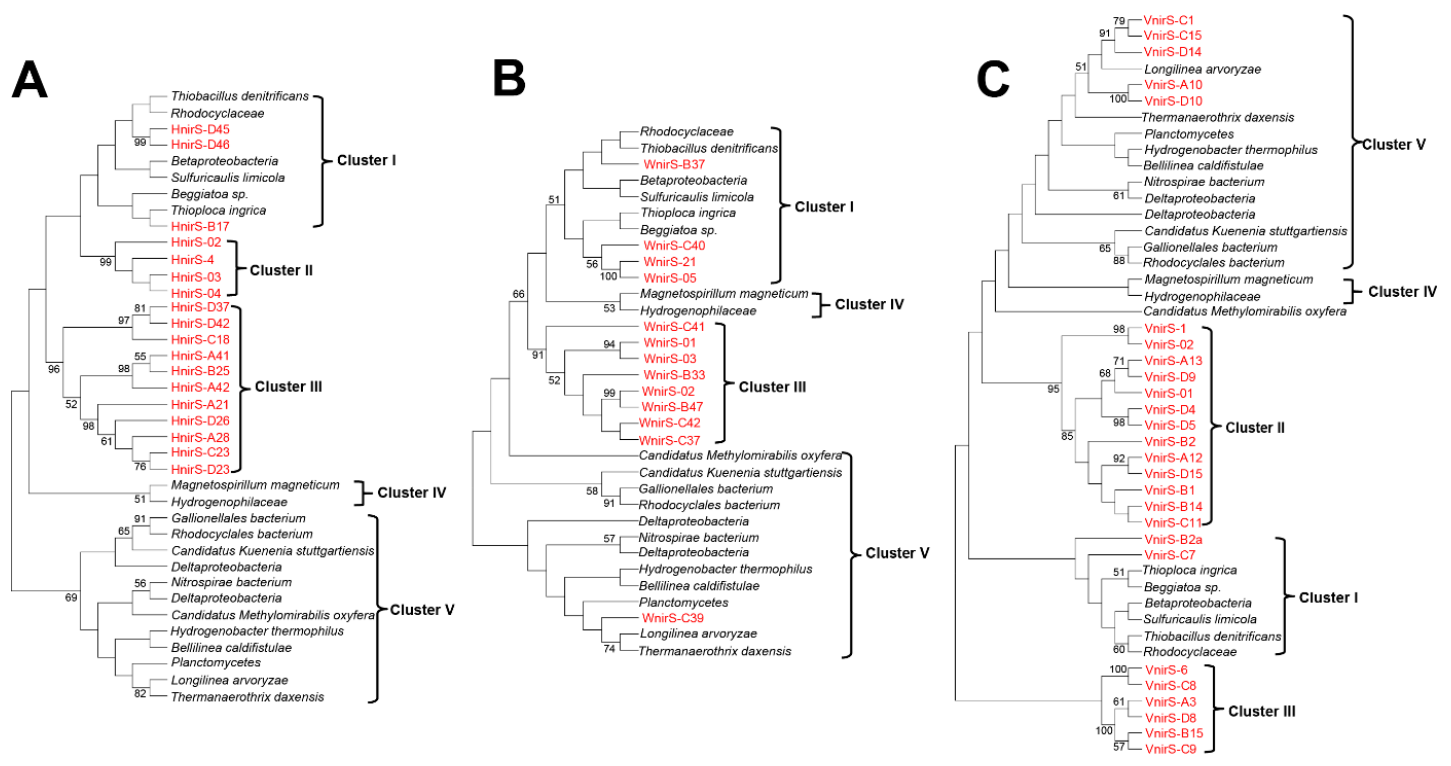

Figure 3. Analysis of nitrite reductase (nirS) gene sequences constructed based on protein sequences using the neighbor-joining method. Sequences from this study are shown in red (A) sample QL-H; (B) sample QL-V; (C) sample SR-W. Other representative nirS from the protein database and GenBank accession numbers are OIO76922 (Hydrogenophilaceae), WP_011386514 (Magnetospirillum magneticum), OFZ68978 (Betaproteobacteria), BAV32586 (Sulfuricaulis limicola), WP_011310590 (Thiobacillus denitrificans), OQY70110 (Rhodocyclaceae), OQY57414 (Beggiatoa sp.), WP_045480144 (Thioploca ingrica), BAE45629 (Hydrogenobacter thermophilus), OGW13923 (Nitrospirae bacterium), OGP39028 (Deltaproteobacteria bacterium), OHB90560 (Planctomycetes), WP_054521542 (Thermanaerothrix daxensis), KPL78197 (Bellilinea caldifistulae), WP_075072401 (Longilinea arvoryzae), OGP16868 (Deltaproteobacteria), CBE69462 (Candidatus Methylomirabilis oxyfera), CAJ74898 (Candidatus Kuenenia stuttgartiensis), OGT00430 (Gallionellales bacterium), and OHC62023 (Rhodocyclales bacterium).

\subsection{Diversity of NirK-Dependent Denitrifying Communities}

Partial nirK gene fragments (ca. 500 b.p.) from the three sample libraries and 150 clones were sequenced. A BLAST analysis revealed that only $31(20 \%)$ of the 150 sequences showed homology to known copper-containing nitrite reductase (nirK) sequences, with 63-96\% nucleotide identity (Figure 4). The deduced amino acid sequences shared 56-99\% identity with NCBI NirK sequences. Consistent with a previous classification [24], the phylogenetic tree grouped all the nirK sequences into five clusters, and predominantly $(86.67 \%)$ within the AOB cluster. Interestingly, only nine clones from sample QL-H fell into nirK, and six clones belonged to one unique protein. Some clones retrieved 
from QL-V and SR-W were grouped with denitrifying bacterial cluster 2, and a single clone from QL-H was grouped into the ammonia-oxidizing archaea (AOA) cluster. These results indicate that bacterial population structures and diversity based on the copper-containing NirK protein differ according to the nitrogen source, which may reflect the occurrence of different 'ecotypes'. Furthermore, a metagenomic qPCR analysis was used to confirm that the copy numbers of nirS retrieved from QL-H, QL-V, and SR-W were $1.15 \times 10^{5}, 1.76 \times 10^{5}$, and $7.15 \times 10^{4}$ gene copies per ng of DNA, respectively, and those of nirK were $3.67 \times 10^{3}, 8.70 \times 10^{3}$, and $1.04 \times 10^{4}$ gene copies per ng of DNA, respectively (Figure 5). The abundances of nirS relative to the overall bacterial population were $0.54 \%, 0.44 \%$, and $0.13 \%$, respectively (Figure 6), and those of nirK were $0.017 \%, 0.022 \%$, and $0.018 \%$, respectively. These results indicate that nirS was more abundant than nirK in all three samples, which is similar to the results observed in some wastewaters [12]. However, the relative abundances of nirK and nirS were clearly significantly lower than those in previously reported samples [8]. These differences could be attributable to the wastewater treatment plants having higher carbon and nitrogen concentrations than our three samples and involving treatment processes that benefit denitrifiers, although our samples were also clearly from habitats that benefit denitrifiers.

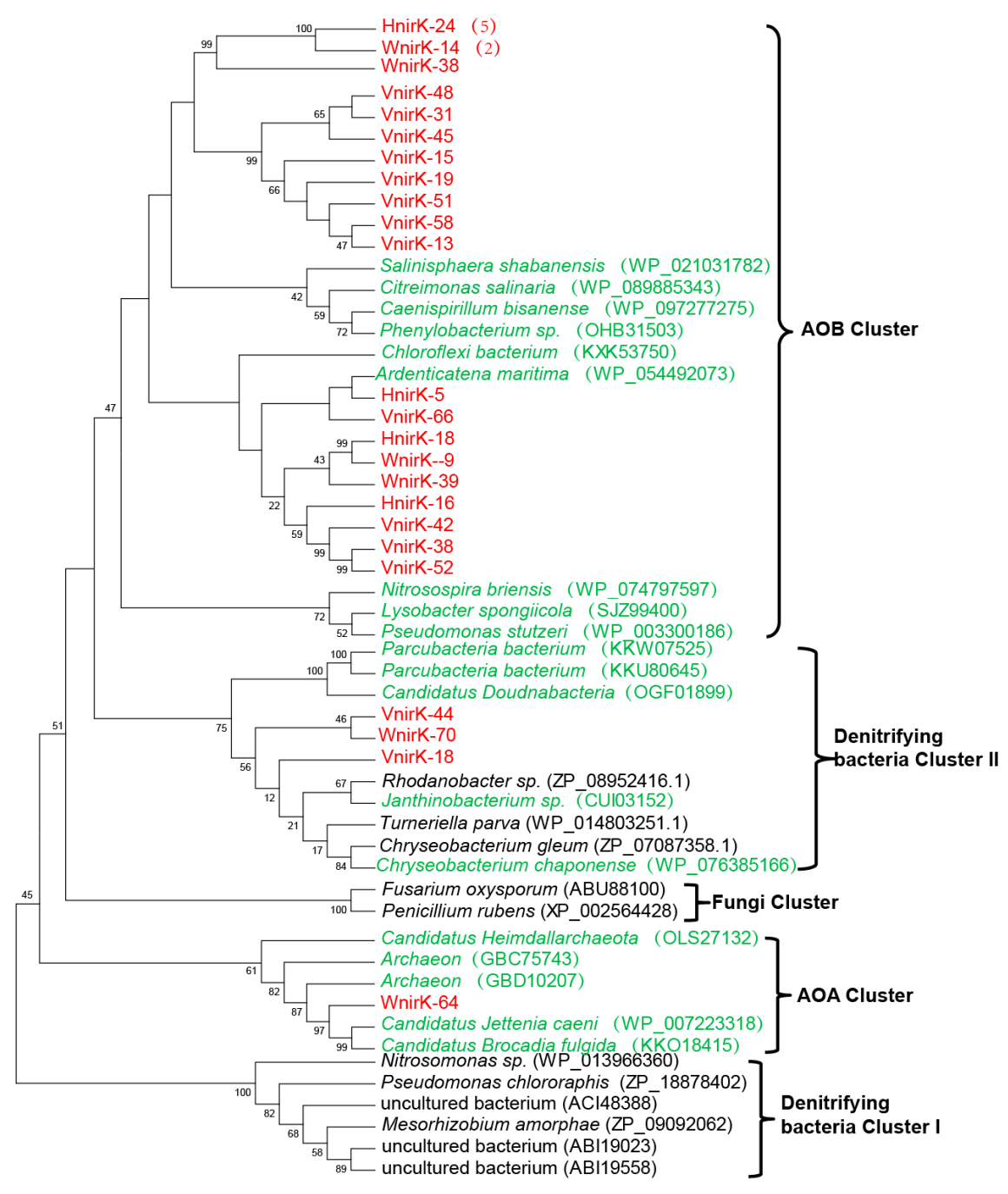

Figure 4. Phylogenetic analysis of NirK gene products (partial, 140 amino acids) from sample QL-H (HnirK), sample QL-V (VnirK), and sample SR-W (WnirK) bacteria (in red). The 19 candidate NirK sequences (in green) were used for designing the NirS gene degenerate primer pair. Database sequences are shown in black, with GenBank accession numbers in parentheses. Roman numerals refer to clusters discussed in the text. Bootstrap values (50\%) for 1000 replicates are shown at the branch points. 


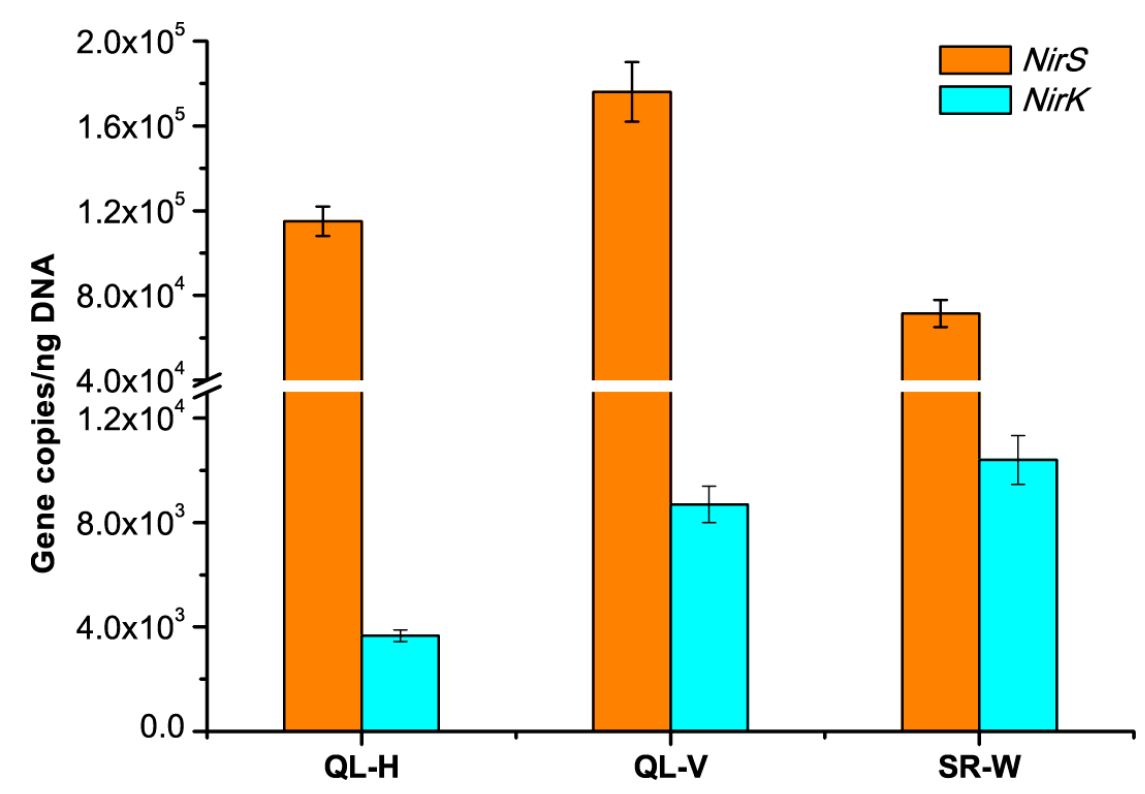

Figure 5. PCR measurements for NirS and NirK gene copy numbers expressed per nanogram of total DNA. Mean values and standard deviations were calculated according to the triplicate assay within a single qPCR setup.

A

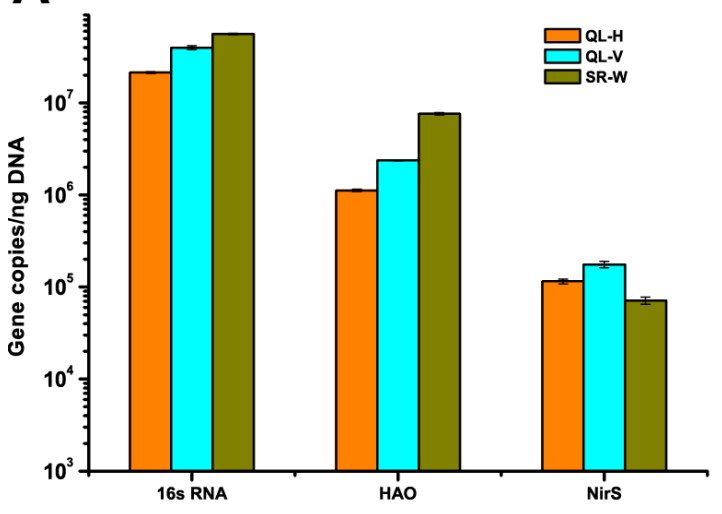

B

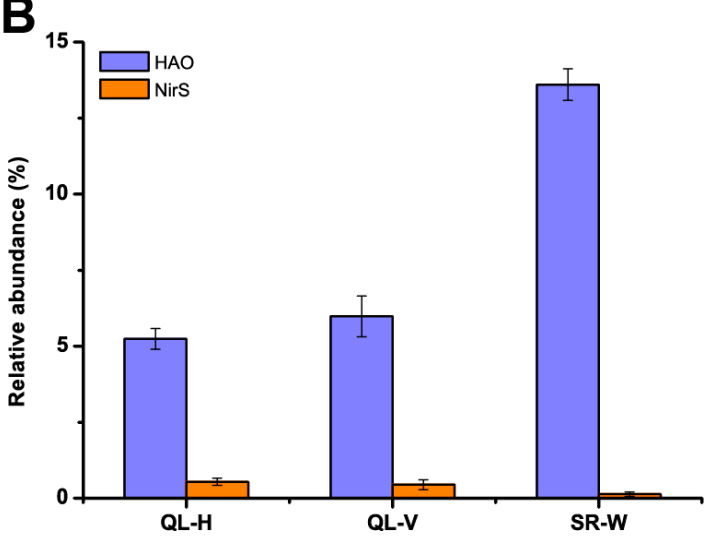

Figure 6. Abundance of bacterial $16 \mathrm{~S}$ rRNA, $H A O$, and NirS genes in different sediment samples. (A) Copy numbers and (B) the relative abundance of $\mathrm{HAO}$ and NirS genes are represented as a percentage of their $16 \mathrm{~S}$ rRNA gene abundance. Mean values and standard deviations were calculated according to the triplicate assay within a single qPCR setup.

\subsection{Abundances of Nitrifying and Denitrifying Bacterial Communities}

Figure $6 \mathrm{~A}$ shows the qPCR results for the $16 \mathrm{~S}$ rDNA, hao, and nirS genes in the three samples. In sludge sample SR-W, with high ammonia and organic contaminant levels, the abundance of $16 \mathrm{~S}$ rDNA was approximately $5.61 \times 10^{7}$ copies/ng DNA, whereas in samples QL-H and QL-V, which were from low-ammonia and low-COD environments, the abundances of $16 \mathrm{~S}$ rDNA were relatively lower (approximately $2.14 \times 10^{7}$ and $3.98 \times 10^{7}$ copies/ng DNA, respectively). It indicates that the SR-W sample had a richer microbial habitat than the others. These differences may be attributable to differences in the compositions of soluble substances and the levels of eutrophication. For example, the SR-W sample had higher levels of carbon, nitrogen, and organic matter than the QL-H and QL-V samples from the lake, and dissolved organic matter is known to be an important factor influencing bacterial community structures [25]. The abundances of the hao and nirS genes of denitrifying bacteria varied significantly among the environmental samples. In sample SR-W, the abundance of the nirS 
gene was below the limit of detection (about $7.15 \times 10^{4}$ copies/ng DNA), whereas in samples QL-H and QL-V, which were taken from high-DO low-ammonium environments, the levels of the nirS gene were $1.15 \times 10^{5}$ and $1.76 \times 10^{5}$ copies/ng DNA, respectively (Figure 6A). Surprisingly, although there were fewer denitrifying bacteria in sludge sample SR-W, the abundance of hao genes was significantly higher (three to sixfold) than in the QL-V and QL-H samples $\left(7.63 \times 10^{6}, 2.38 \times 10^{6}\right.$, and $1.12 \times 10^{6}$ copies/ng DNA, respectively).

\section{Discussion}

In this study, gene-specific metagenomic PCR and qPCR amplification were used to analyze the environmental factors that influence the microbial community and nitrogen nutrition-related bacterium from three environmental sediment samples with distinct nitrogen source gradients.

Phylogenetic analysis did not show distinct distribution differences in the communities among the nutrient-associated environments. Thus, we further analyzed the overall compositions and distribution of bacterial subdivisions. For example, in sample QL-H, the Proteobacteria were significantly affiliated with the Gammaproteobacteria $(21.21 \%)$ and Betaproteobacteria $(18.19 \%)$ clusters, whereas in the SR-W sample, they were more commonly associated with the Betaproteobacteria clusters, which were distributed on two distinct phylogenetic branches, and only one clone was clustered within the Alphaproteobacteria (Figure 1). In contrast, Proteobacteria was not the most abundant phylum in sample QL-V, and all the members were affiliated with the Betaproteobacteria class on distinct phylogenetic branches (Figure 1). The native composition of the microbial community in the QL-H sample was dominated by uncultured bacteria $(39.30 \%)$ and the Cytophagales-Sphingobacteria group $(18.18 \%)$. Interestingly, the community diversity in sample QL-V, which was collected in a neighboring village, displayed no relatively concentrated phyla, and uncultured bacteria dominated the clone library. Few clones from the sample QL-V sequences were affiliated with Aquabacterium, Enterovibrio, or Verrucomicrobia. The sequences from the SR-W sample dataset were distinctly different from those of the other samples, and were assigned to Aquabacterium (25\%), Betaproteobacteria (25\%), Bacteroidetes, and uncultured bacteria. Members of the beta class of the Proteobacteria were well represented under all the environmental conditions, whereas the abundances of the other classes differed significantly. The predominance of Proteobacteria in water samples of different quality suggests that the distribution of structurally simple prokaryotic consortia along microscale biogeochemical gradients is a unique and effective strategy by which bacteria meet their requirements for growth in otherwise inhospitable environments [26]. We also observed a clear difference in the community compositions in the SR-W and QL-H samples, which varied according to their levels of inorganic nitrogen. The genus Aquabacterium was overrepresented in the high ammonia-nitrogen water because they have an efficient nitrate-dependent $\mathrm{Fe}$ (II) oxidation capacity, using nitrate as an electron acceptor [27]. Overall, the shifts in community composition along the gradients of dissolved organic carbon (DOC) and inorganic contaminants are similar to the patterns of growth efficiency and growth rates [28]. These results suggest that bacterial growth efficiency, abundance, and community composition are presumably constrained by the substrate concentrations of nitrogen compounds in most natural waters, with the possible exception of the most oligotrophic environments [29].

Functional biomarkers related to the physiology of specific bacteria, such as genes encoding unique enzymes involved in their specific biochemical metabolism [30], could offer a better alternative for exploring the diversity and population characteristics of bacterial communities. Furthermore, all the Hao proteins of the AOB clustered with Nitrosomonas, whereas no sequence clustered with the Nitrosospira lineage (Figure 2). Similar studies have reported that Nitrosomonas predominates in tannery wastewater treatment plants [10-12]. However, unlike the predominance of N. europaea in various copiotrophic samples, such as laboratory-scale anaerobic ammonium-oxidizing reactors [31], full-scale modified Ludzack-Ettinger process wastewater treatment plants [11], and pilot-scale batch nitrifying reactors [32], none of our recovered clones clustered with N. europaea. Instead, N. oligotropha and N. ureae dominated our three samples. Previous studies have demonstrated that the apparent affinity of 
$N$. oligotropha-like AOB for low ammonium may allow them to outcompete the more copiotrophic $N$. europaea-like AOB strains [33]. In this work, it is clear that the Hao-dependent AOB species differed in the three water samples in parallel with the dramatic changes in ammonia nitrogen (Table 1). The three distinct ammonium-graduated sites QL-H (undetectable ammonium), QL-V (16.34 mg/L), and SR-W $(93.43 \mathrm{mg} / \mathrm{L})$ AOB were approximately affiliated to $N$. ureae with $2 \%, 13 \%$, and $80 \%$, respectively. The high concentration of ammonia in the influent of SR-W may have contributed to the predominance of clones related to the $N$. ureae lineage, which was consistent with previous studies that the $N$. ureae cluster was the predominant $\mathrm{AOB}$, was commonly found in environments rich in ammonium [34], and can tolerate up to $200 \mathrm{mM}$ of ammonium [35]. In contrast, $N$. oligotropha already dominated in the AOB of the QL-H sample $(>90 \%)$, and also made up more than $65 \%$ in the QL-V sample and small amounts $(\sim 8 \%)$ in the RS-W sample. The N. oligotropha of AOB species are often found in freshwater due to its lower the $K s$ and $K m$ values for ammonia by an order of magnitude more than N. europaea, N. eutropha, or their close relatives [35], allowing them to grow at low ammonium concentrations [36]. Meanwhile, N. oligotropha can also adapt to grow at higher ammonium availability, but with a longer lag phase [36]. Our data show that in oligotrophic environments, carbon and nitrogen are probably important determinants of bacterial community composition and structure, and possibly shifting toward supporting microbial groups. Therefore, the distinct and characteristic $N$. oligotropha population structures in the three environments can be attributed to the sharp differences in the nitrogen concentrations, particularly because in oligotrophic environments, representatives of $N$. oligotropha may outcompete other species.

The newly identified NirS sequences were predominantly affiliated with unknown clusters II and III, and occasionally within unknown cluster I, and the distributions of these subclusters clearly differed. The sequences recovered from the oligotrophic environment of QL-H fell in unknown cluster II (Figure 3A), sharing 75-89\% protein sequence identity with other reported sequences. This cluster consisted of four unique NirS sequences and 13 clones that were most closely related to the class Gammaproteobacteria, including the family Xanthomonadaceae (80\%) and Marinobacter spp. (79\%). A previous study demonstrated that members of Xanthomonadaceae could function as autotrophic denitrifiers in a pyrite-fed denitrifying reactor [37], and the Marinobacter NirS protein has an important role as a biological marker of microbial degradation in aquatic environments [38]. The grouping of the QL-H sequences with unique cluster II appears to be closely associated with the oligotrophic status of the lake environment, although they formed a new clade within the dominant group. This is consistent with the 16S rRNA results, which showed that the Gammaproteobacteria had high specificity and were highly abundant in the QL-H sample, which was collected from an environment with low ammonium concentration (Figure 1). This new phylogenetic group has rarely been detected in other high ammonia-nitrogen aquatic environments or lakes, and was never retrieved in previous studies of Lake Bourget. Our high success rate with metagenomic PCR screening indicates that this oligotrophic lake is a unique reservoir of diverse and novel nitrite reductase family members, which may represent novel denitrifying bacteria with key roles in limnic biogeochemical nitrogen cycling. In contrast, the sequences recovered from SR-W were predominantly affiliated with unknown clusters I (30.43\%) and III (67.39\%) (Figure 3B), sharing $89-100 \%$ protein sequence identity with previously reported sequences. A single clone grouped in unknown cluster $\mathrm{V}$, sharing $83 \%$ protein sequence identity with Chloroflexi bacterium [39], which is an autotrophic denitrifier whose performance seems to benefit from nitrate removal. Similarly, members of unique cluster III were also observed in the QL-V sample $(20 \%)$, together with members of the two dominant unique clusters (I and III) (Figure 3C). The sequences in cluster II shared $73-88 \%$ protein sequence identity with sequences retrieved from activated sludge in municipal wastewater and coastal wetlands [9]. Five clones were grouped in cluster V, sharing $77-89 \%$ protein sequence identity with sequences retrieved from terrestrial subsurface sediments [40].

These nir-based phylogenetic trees revealed important patterns and distributions in the denitrifying community in three different environments providing different nitrogen sources (Figures 3 and 4). The majority of nirS clones were found in subclusters within the major clusters formed by unknown 
denitrifying bacteria, and did not cluster with any known denitrifying bacteria. In contrast, the clones retrieved from the higher-nitrogen QH-V and SR-W samples clustered with more known denitrifying bacteria than those from QL-H. The distribution and diversity of nirK-dependent denitrifiers in the various habitats were similar to those of the nirS-dependent denitrifiers. This indicates that the three sediments contained unique denitrifiers, which were unknown among previously reported denitrifiers. These results suggest that the distributions and abundances of some denitrifying bacteria correlated with the bioavailability of nitrogen and carbon, whereas the distributions of others (e.g., nirS clusters I and $\mathrm{V}$, and nirK cluster AOB) correlated negatively with the carbon and nitrogen sources. Undeniably, some unique clusters were not detected in the two most extreme environments (the most and least eutrophic) from which our samples were drawn, suggesting that they may only occur at very low levels. Previous studies have shown that long-term supplementation with organic fertilizer or the addition of root-derived carbon only slightly affected the denitrifier community structure [1]. Therefore, the distribution of denitrifier clusters may reflect their adaptation to the various intermediate or hybrid environmental conditions they encounter [23,41].

Notably, the qPCR analysis showed that the hao gene abundances in all three samples were significantly higher than the nirS gene abundances by orders of magnitude. Compared with the $16 \mathrm{~S}$ rDNA detected in the environmental habitats, the relative abundances of the hao gene in samples QL-H, QL-V, and SR-W were $5.23 \%, 5.97 \%$, and $13.60 \%$, respectively, whereas the relative abundances of the nirS gene were $0.54 \%, 0.44 \%$, and $0.13 \%$, respectively (Figure $6 \mathrm{~B}$ ). The highest abundance of the hao gene was detected in sample SR-W from a river containing domestic sewage, where the higher ammonia nitrogen and eutrophication caused nitrifying bacteria to be the dominant bacteria. Notably, the numbers of denitrifying bacterial nirS genes were lower than the free-living ones at the closest-to-lake site. Previous studies have shown that total inorganic nitrogen, ammonium, and the organic content are important factors affecting community structures, denitrification rates, and Nir abundance of environmental microbes [25]. With the high concentration of ammonium in the SR-W sample, the accumulation of nitrate and nitrite was significantly higher than in the samples of lake water. Nitrate and nitrite also strongly affect denitrification richness [42], which is consistent with the results for the n-damo enrichments [43]. Environmental factors correlated differently and even oppositely with the 16S rDNA and denitrifier-specific sequences in the three samples, indicating that their influence on the nitrifying and denitrifying community structures is very complex.

Together, we detected nitrifying and denitrifying bacteria in three complex and contrasting environments with gene-specific metagenomic PCR amplification of the unique functional marker genes hao, nirS, and nirK. This provided comprehensive evidence that the microbial community structures of nitrifiers and denitrifiers are influenced by the environmental nitrogen status. The hao genes of the majority of nitrifying bacteria retrieved and the nirK and nirS genes of the denitrifying bacteria were affiliated with those of different genera of uncultured bacteria, but distantly related to the genes of known nitrifiers and denitrifiers, respectively. The high diversities and abundances observed in the Hao and Nir phylogenies suggest high interspecies variety in the three different environments (which differed sharply in their nitrogen and organic carbon status), which caused pronounced shifts in the nitrifying and denitrifying populations. The characteristic nitrifier and denitrifier population structures differed according to the nitrogen source, which was reflected in the predominant potential nitrifying and denitrifying genera present, and especially in the many redundant clones that were present, which may contribute greatly to the nitrogen metabolism in these unique environments. These results extend our knowledge of the biology and diversity of nitrifiers and denitrifiers in natural lakes and polluted rivers, and may have practical utility in the efficient restoration of rivers and lakes. However, the high diversity of marker genes and the changing patterns of bacterial dominance do not really reflect their contribution to nitrogen cycling. Novel experimental approaches such as transcriptome, proteomics, and metabolomics [44] are required to detect active nitrifiers and denitrifiers, to better understand their roles and the mechanisms they use in nitrogen cycling and environmental bioremediation. 
Author Contributions: P.J. and Z.Z. wrote the manuscript. Sample collection, processing and analysis were carried out by R.Z., Y.C., J.Q. and Z.C.

Funding: This research was supported by the National Natural Science Foundation of China (Grant 31700078 and Grant 31270724), the Scientific Research Foundation for Talent program of Zhejiang Agricultural and Forestry University (W20170029), Distinguished Scholars of Zhejiang Agricultural and Forestry University (2014FR064).

Conflicts of Interest: The authors declare no competing financial interests.

\section{References}

1. Philippot, L.; Hallin, S.; Schloter, M. Ecology of denitrifying prokaryotes in agricultural soil. Adv. Agron. 2007, 96, 249-305.

2. Arrigo, K.R. Marine microorganisms and global nutrient cycles. Nature 2005, 437, 349-355. [CrossRef] [PubMed]

3. Xia, J.; Wan, S. Independent effects of warming and nitrogen addition on plant phenology in the Inner Mongolian steppe. Ann. Bot. Lond. 2013, 111, 1207-1217. [CrossRef] [PubMed]

4. Xie, J.; Chen, J.; Sun, G.E.; Chu, H.; Noormets, A.; Ouyang, Z.; Ranjeet, J.; Wan, S.; Guan, W. Long-term variability and environmental control of the carbon cycle in an oak-dominated temperate forest. For. Ecol. Manag. 2014, 313, 319-328. [CrossRef]

5. Lozupone, C.; Knight, R. UniFrac: a new phylogenetic method for comparing microbial communities. Appl. Environ. Microb. 2005, 71, 8228-8235. [CrossRef] [PubMed]

6. Chen, W.; Hao, L.; Zhang, Q.; Dai, S. Effect of nitrite on growth and microcystins production of Microcystis aeruginosa PCC7806. J. Appl. Phycol. 2011, 23, 665-671. [CrossRef]

7. Zhang, Y.; Xie, X.; Jiao, N.; Hsiao, S.S.Y.; Kao, S.J. Diversity and distribution of amoA-type nitrifying and nirS-type denitrifying microbial communities in the Yangtze River estuary. Biogeosciences 2014, 12, 2131-2145. [CrossRef]

8. Oakley, B.B.; Francis, C.A.; Roberts, K.J.; Fuchsman, C.A.; Srinivasan, S.; Staley, J.T. Analysis of nitrite reductase (nirK and nirS) genes and cultivation reveal depauperate community of denitrifying bacteria in the Black Sea suboxic zone. Environ. Microbiol. 2007, 9, 118-130. [CrossRef] [PubMed]

9. Gao, J.; Hou, L.; Zheng, Y.; Liu, M.; Yin, G.; Li, X.; Lin, X.; Yu, C.; Wang, R.; Jiang, X. Nirs-Encoding denitrifier community composition, distribution, and abundance along the coastal wetlands of China. Appl. Microbiol. Biotechnol. 2016, 100, 8573-8582. [CrossRef]

10. Zhang, T.; Ye, L.; Tong, A.H.; Shao, M.F.; Lok, S. Ammonia-oxidizing archaea and ammonia-oxidizing bacteria in six full-scale wastewater treatment bioreactors. Appl. Microbiol. Biotechnol. 2011, 91, 1215-1225. [CrossRef]

11. Figuerola, E.L.M.; Erijman, L. Diversity of nitrifying bacteria in a full-scale petroleum refinery wastewater treatment plant experiencing unstable nitrification. J. Hazard. Mater. 2010, 181, 281-288. [CrossRef] [PubMed]

12. Wang, Z.; Zhang, X.X.; Lu, X.; Liu, B.; Li, Y.; Long, C.; Li, A. Abundance and diversity of bacterial nitrifiers and denitrifiers and their functional genes in tannery wastewater treatment plants revealed by high-throughput sequencing. PLoS ONE 2014, 9, e113603. [CrossRef] [PubMed]

13. Liu, W.; Bao, Q.; Jirimutu; Qing, M.; Siriguleng; Chen, X.; Sun, T.; Li, M.; Zhang, J.; Yu, J. Isolation and identification of lactic acid bacteria from Tarag in Eastern Inner Mongolia of China by 16S rRNA sequences and DGGE analysis. Microbiol. Res. 2012, 167, 110-115. [CrossRef] [PubMed]

14. Lindström, E.S.; Agterveld, K.V.; Zwart, G. Distribution of typical freshwater bacterial groups is associated with ph, temperature, and lake water retention time. Appl. Environ. Microb. 2005, 71, 8201-8206. [CrossRef] [PubMed]

15. Zhao, C.; Fu, S.; Mathew, R.P.; Lawrence, K.S.; Feng, Y. Soil microbial community structure and activity in a 100-year-old fertilization and crop rotation experiment. J. Plant Ecol. 2015, 60, 741-747. [CrossRef]

16. Iwai, S.; Chai, B.; Sul, W.J.; Cole, J.R.; Hashsham, S.A.; Tiedje, J.M. Gene-targeted-metagenomics reveals extensive diversity of aromatic dioxygenase genes in the environment. ISME J. 2010, 4, 279-285. [CrossRef] [PubMed]

17. Jin, P.; Pei, X.; Du, P.; Yin, X.; Xiong, X.; Wu, H.; Zhou, X.; Wang, Q. Overexpression and characterization of a new organic solvent-tolerant esterase derived from soil metagenomic DNA. Bioresour. Technol. 2012, 116, 234-240. [CrossRef] 
18. Montgomery, H.A.C.; Thom, N.S.; Cockburn, A. Determination of dissolved oxygen by the winkler method and the solubility of oxygen in pure water and sea water. J. Chem. Technol. Biot. 2010, 14, 280-296. [CrossRef]

19. Tamura, K.; Peterson, D.; Peterson, N.; Stecher, G.; Nei, M.; Kumar, S. MEGA5: molecular evolutionary genetics analysis using maximum likelihood, evolutionary distance and maximum parsimony methods. Mol. Biol. Evol. 2011, 28, 2731-2739. [CrossRef]

20. Regan, J.M.; Harrington, G.W.; Noguera, D.R. Ammonia- and nitrite-oxidizing bacterial communities in a pilot-scale chloraminated drinking water distribution system. Appl. Environ. Microb. 2002, 68, 73-81. [CrossRef]

21. Ball, P.N.; Mackenzie, M.D.; Deluca, T.H.; Holben, W.E. Wildfire and charcoal enhance nitrification and ammonium-oxidizing bacterial abundance in dry montane forest soils. J. Environ. Qual. 2010, 39, 1243-1253. [CrossRef]

22. Kartal, B.; Maalcke, W.J.; Almeida, N.M.D.; Cirpus, I.; Gloerich, J.; Geerts, W.; Camp, H.J.M.O.D.; Harhangi, H.R.; Janssenmegens, E.M.; Francoijs, K.J. Molecular mechanism of anaerobic ammonium oxidation. Nature 2011, 479, 127-130. [CrossRef]

23. Dang, H.; Wang, C.; Li, J.; Li, T.; Tian, F.; Jin, W.; Ding, Y.; Zhang, Z. Diversity and distribution of sediment nirS-encoding bacterial assemblages in response to environmental gradients in the eutrophied Jiaozhou Bay, China. Microb. Ecol. 2010, 58, 161-169. [CrossRef]

24. Long, A.; Song, B.; Fridey, K.; Silva, A. Detection and diversity of copper containing nitrite reductase genes (nirK) in prokaryotic and fungal communities of agricultural soils. FEMS Microbiol. Ecol. 2015, 91, 1-9. [CrossRef]

25. Shen, L.D.; Liu, S.; Zhu, Q.; Li, X.Y.; Cai, C.; Cheng, D.Q.; Lou, L.P.; Xu, X.Y.; Zheng, P.; Hu, B.L. Distribution and diversity of nitrite-dependent anaerobic methane-oxidising bacteria in the sediments of the Qiantang River. Microb. Ecol. 2014, 67, 341-349. [CrossRef]

26. Gao, D.; Wang, X.; Fu, S.; Zhao, J. Legume plants enhance the resistance of soil to ecosystem disturbance. Front. Plant Sci. 2017, 8, 1295. [CrossRef]

27. Zhang, X.; Li, A.; Szewzyk, U.; Fang, M. Improvement of biological nitrogen removal with nitrate-dependent Fe(II) oxidation bacterium Aquabacterium parvum B6 in an up-flow bioreactor for wastewater treatment. Bioresour. Technol. 2016, 219, 624-631. [CrossRef]

28. Eiler, A.; Langenheder, S.; Bertilsson, S.; Tranvik, L.J. Heterotrophic bacterial growth efficiency and community structure at different natural organic carbon concentrations. Appl. Environ. Microb. 2003, 69, 3701-3709. [CrossRef]

29. Fernándezgómez, B.; Richter, M.; Schüler, M.; Pinhassi, J.; Acinas, S.G.; González, J.M.; Pedrósalió, C. Ecology of marine Bacteroidetes: a comparative genomics approach. ISME J. 2013, 7, 1026-1037. [CrossRef]

30. Li, M.; Ford, T.; Li, X.; Gu, J.D. Cytochrome cd1-containing nitrite reductase encoding gene nirS as a new functional biomarker for detection of anaerobic ammonium oxidizing (Anammox) bacteria. Environ. Sci. Technol. 2011, 45, 3547-3553. [CrossRef]

31. Quan, Z.X.; Rhee, S.K.; Zuo, J.E.; Yang, Y.; Bae, J.W.; Park, J.R.; Lee, S.T.; Park, Y.H. Diversity of ammonium-oxidizing bacteria in a granular sludge anaerobic ammonium-oxidizing (anammox) reactor. Environ. Microbiol. 2008, 10, 3130-3139. [CrossRef]

32. Gieseke, A.; Bjerrum, L.; Wagner, M.; Amann, R. Structure and activity of multiple nitrifying bacterial populations co-existing in a biofilm. Environ. Microbiol. 2003, 5, 355-369. [CrossRef]

33. Gieseke, A.; Purkhold, U.; Wagner, M.; Amann, R.; Schramm, A. Community structure and activity dynamics of nitrifying bacteria in a phosphate-removing biofilm. Appl. Environ. Microb. 2001,67,1351-1362. [CrossRef]

34. Allen, J.G.; Beutel, M.W.; Call, D.R.; Fischer, A.M. Effects of oxygenation on ammonia oxidation potential and microbial diversity in sediment from surface-flow wetland mesocosms. Bioresour. Technol. 2010, 101, 1389-1392. [CrossRef]

35. Gabriele, S.; Barbara, B.; Petra, D.; Gabriele, R.; Koops, H.P. The ammonia-oxidizing nitrifying population of the River Elbe estuary. FEMS Microbiol. Ecol. 2010, 17, 177-186.

36. Bollmann, A.; Laanbroek, H.J. Continuous culture enrichments of ammonia-oxidizing bacteria at low ammonium concentrations. FEMS Microbiol. Ecol. 2001, 37, 211-221. [CrossRef]

37. Gorshkova, N.M.; Ivanova, E.P.; Sergeev, A.F.; Zhukova, N.V.; Alexeeva, Y.; Wright, J.P.; Nicolau, D.V.; Mikhailov, V.V.; Christen, R. Marinobacter excellens sp. nov., isolated from sediments of the Sea of Japan. Int. J. Syst. Evol. Micr. 2003, 53, 2073-2078. [CrossRef] [PubMed] 
38. Torrentó, C.; Urmeneta, J.; Otero, N.; Soler, A.; Viñas, M.; Cama, J. Enhanced denitrification in groundwater and sediments from a nitrate-contaminated aquifer after addition of pyrite. Chem. Geol. 2011, 287, 90-101. [CrossRef]

39. Xiao, Y.; Zheng, Y.; Wu, S.; Yang, Z.H.; Zhao, F. Bacterial community structure of autotrophic denitrification biocathode by 454 pyrosequencing of the 16s rrna gene. Microb. Ecol. 2015, 69, 492-499. [CrossRef]

40. Hernsdorf, A.W.; Amano, Y.; Miyakawa, K.; Ise, K.; Suzuki, Y.; Anantharaman, K.; Probst, A.; Burstein, D.; Thomas, B.C.; Banfield, J.F. Potential for microbial $\mathrm{H}_{2}$ and metal transformations associated with novel bacteria and archaea in deep terrestrial subsurface sediments. ISME J. 2017, 11, 1915-1929. [CrossRef]

41. Kim, Y.C.; Gao, C.; Zheng, Y.; He, X.H.; Yang, W.; Chen, L.; Wan, S.Q.; Guo, L.D. Arbuscular mycorrhizal fungal community response to warming and nitrogen addition in a semiarid steppe ecosystem. Mycorrhiza 2015, 25, 267. [CrossRef] [PubMed]

42. Chen, W.; Hao, L. Intracellular nitrite accumulation: The cause of growth inhibition of Microcystis aeruginosa exposure to high nitrite level. Phycol. Res. 2015, 63, 197-201. [CrossRef]

43. Mosier, A.C.; Francis, C.A. Denitrifier abundance and activity across the San Francisco Bay estuary. Environ. Microbiol. Rep. 2010, 2, 667-676. [CrossRef]

44. Nogales, B.; Timmis, K.; Nedwell, D.; Osborn, A. Detection and diversity of expressed denitrification genes in estuarine sediments after reverse transcription-PCR amplification from mRNA. Appl. Environ. Microb. 2002, 68, 5017-5025. [CrossRef] [PubMed]

(C) 2019 by the authors. Licensee MDPI, Basel, Switzerland. This article is an open access article distributed under the terms and conditions of the Creative Commons Attribution (CC BY) license (http://creativecommons.org/licenses/by/4.0/). 